\title{
Exudative Polymorphous Vitelliform Retinopathy: Importance of Early Recognition of the Condition in Patients with Metastatic Melanoma
}

\author{
Norah Lincoff $\cdot$ Muhammad Nadeem · Zilfah Younus • \\ Charles E. Thirkill
}

To view enhanced content go to www.ophthalmology-open.com

Received: November 16, 2015 / Published online: February 18, 2016

(c) The Author(s) 2016. This article is published with open access at Springerlink.com

\begin{abstract}
Introduction: Because of the advent of monoclonal antibodies in the treatment of metastatic melanoma, patients with this disease are surviving longer. Early recognition of the disease has therefore become even more important. Case report: We present a patient with vitelliform maculopathy, a paraneoplastic retinal maculopathy that is under-recognized. Clinically the retinal findings of serous detachments and pigmentary macular changes are remarkable, while at the same time these patients have surprisingly very few symptoms. This is in contrast to patients who develop melanoma associated retinopathy (MAR) who are very symptomatic early in the disease, but with more subtle retinal findings.
\end{abstract}

N. Lincoff $(\varangle) \cdot$ M. Nadeem · Z. Younus

Buffalo General Medical Center, State University of

New York, Buffalo, 100 High Street, Buffalo, NY

14203, USA

e-mail: lincoff@buffalo.edu

C. E. Thirkill

Ocular Immunology Lab 1220, University of

California, Davis, U. C. Davis, Davis, CA, USA
Conclusion: Monoclonal antibody treatment is changing the survival rates in metastatic disease making early diagnosis even more important. Exudative polymorphous vitelliform maculopathy (EPVM) needs to be recognized early to avoid delay in diagnosis of metastatic disease.

Keywords: Exudative; Maculae; Metastatic melanoma; Retinopathy; Paraneoplastic; Retinal maculopathy; Vitelliform

\section{INTRODUCTION}

Due to the advent of monoclonal antibody agents in the treatment of metastatic melanoma, patients with this disease are surviving longer [1]. Early recognition of the disease has therefore become even more important. We present a patient with vitelliform maculopathy, a paraneoplastic retinal maculopathy that is under-recognized. Clinically the retinal findings of serous detachments and macular pigmentary changes are remarkable, while at the same time these patients have surprisingly very few symptoms. This is in contrast to patients who develop 
(a)

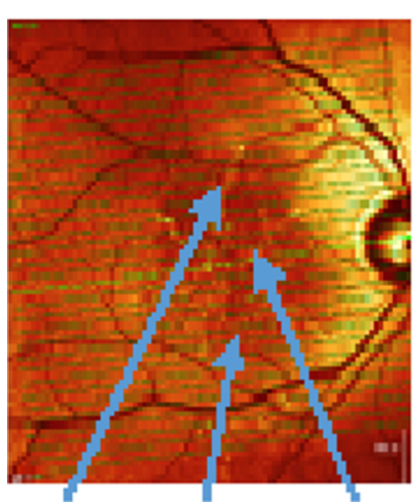

(b)

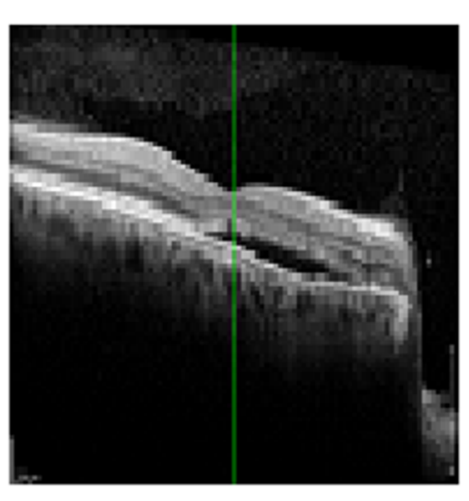

(c)

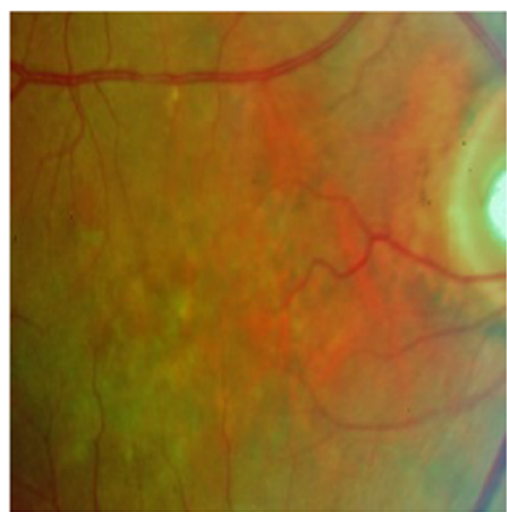

Fig. 1 a Pigmentary and atrophic changes on OCT, $\mathbf{b}$ serous detachment on OCT in the pre-treatment right eye, and c pigmentary and atrophic changes on color fundus photograph. OCT optical coherence tomography

(a)

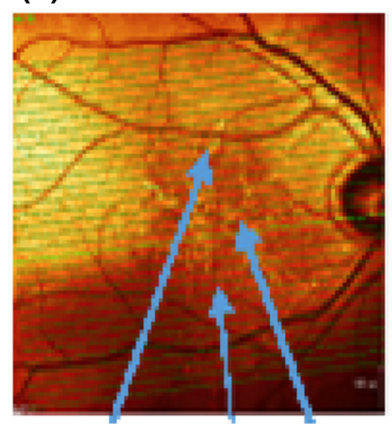

(b)

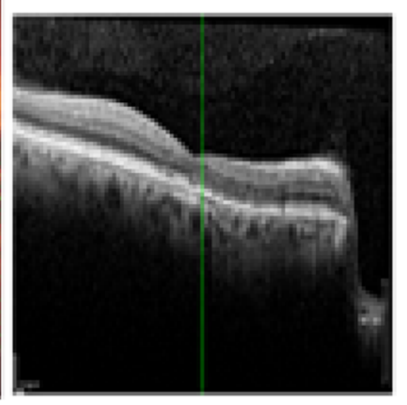

Fig. 2 a Pigmentary and atrophic changes on OCT, and b post-treatment resolution of serous detachment on OCT in the right eye. OCT optical coherence tomography

melanoma associated retinopathy (MAR) who are very symptomatic early in the disease, but with more subtle retinal findings.

Informed consent was obtained from all patients for being included in the study.

\section{CASE REPORT}

A 65-year-old male being worked up for metastatic disease was found to have multiple exudative vitelliform lesions in both maculae (Figs. 1, 3). These lesions are found in patients with metastatic melanoma. Biopsy of an occipital lesion confirmed melanoma (Fig. 5a).
A positron emission tomography (PET) scan also showed lesions in the lung, axilla and gallbladder (Fig. 5b). No choroidal masses were found. Through radiology, the gallbladder was felt to be the site of primary malignancy. The metastatic lesions, as well as the exudative macular lesions both improved with monoclonal antibody (ipilimumab) treatment (Figs. 2, 4). One year following treatment there was no sign of recurrence of disease. The patient has been followed up every 3 months since using PET scans.

The patient initially had symptoms of dizziness and the feeling that he was missing characters to the left of fixation for seconds at a time while reading. Following resection of his $\mathrm{R}$ occipital mass, and treatment with chemotherapy his symptoms slowly improved over a 6-month period. He denied ever having significant blurring, any types of photopsias, or trouble with light/dark adaptation. His vision was correctable to 20/25 in his right eye and 20/20 in his left eye. His color vision and pupils were normal. Visual fields by confrontation were normal while Humphrey Visual Field test (HVF) 24-2 revealed a small central L hemianopsia attributable to his occipital 
(a)

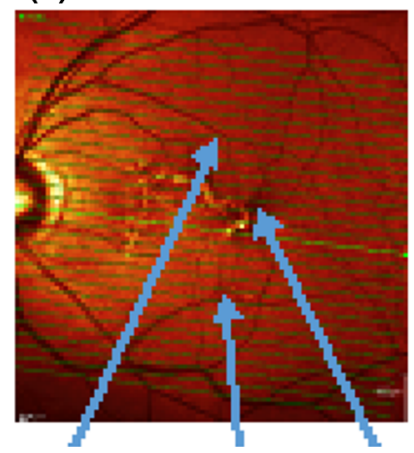

(b)

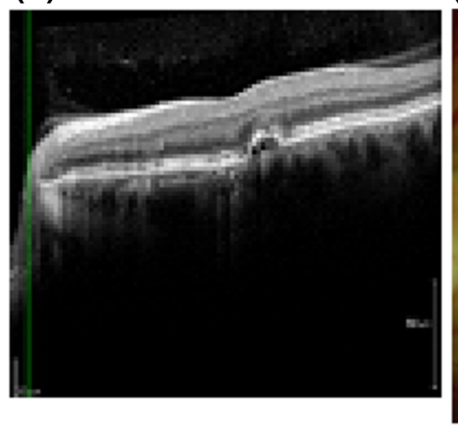

(c)

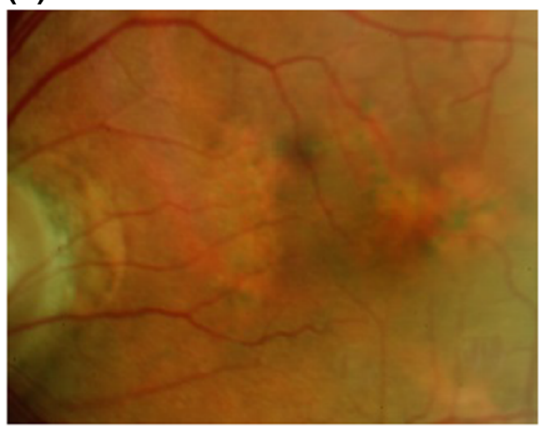

Fig. 3 a Pigmentary and atrophic changes on OCT, $\mathbf{b}$ serous detachment on OCT on pre-treatment left eye, and c pigmentary and atrophic changes on Fundus photograph. $O C T$ optical coherence tomography

(a)

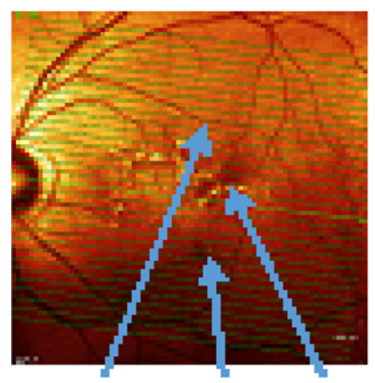

(b)

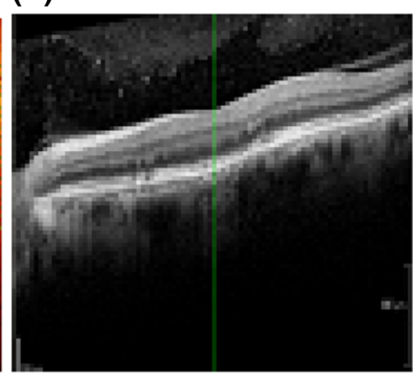

Fig. 4 a Pigmentary and atrophic changes on OCT, and b post-treatment resolution of serous detachment on OCT in the left eye. OCT optical coherence tomography

disease. His intraocular pressure was $13 \mathrm{mmHg}$ in both eyes. Slit lamp examination was unremarkable. Funduscopic examination revealed crisscross pigmentary changes in both maculae with muting of the foveal reflex. Both optic nerves and peripheral retinas were normal. Motility examination was normal. Heidelberg optical coherence tomography (OCT) revealed multiple shallow retinal pigment epithelial (RPE) detachments with significant disruption of the outer retinal layers with bright vitelliform lesions throughout the areas of detachment (Figs. 1, 3 ). The areas of detachment on OCT responded to treatment over a 4-month period without recurrence (Figs. 2, 4).
The patient presented with significant retinal RPE damage as seen in the fundus photographs (Figs. 1c, 3c). Use of the OCT helped delineate the numerous smaller shallow serous detachments in each eye, which is not typical of patients with Best's Disease or central serous retinopathy (CSR). Patients with CSR and Best's Disease typically have one central larger area of detachment making them more acutely symptomatic. In Best's disease there is usually also lipofuscin accumulation centrally causing the "egg yolk" appearance. The crisscross and linear pattern RPE changes seen in our patient with EPVM are also atypical for Best's disease and CSR. The OCT helped monitor a response to the patients' systemic treatment without the need of angiography. Fluorescein angiography revealed the expected area of RPE damage with minimal staining and no leakage (Figs. 6, 7). Fundus autofluorescence imaging delineates the linear yellowish lipofuscinoid deposits and areas of old detachment, and small areas of pigment epithelial atrophy (Fig. 8).

\section{DISCUSSION}

In the 1990s the 5 -year survival rate of stage 4 melanoma to the brain was less than $4 \%$, until the advent of new therapies including 
(a)

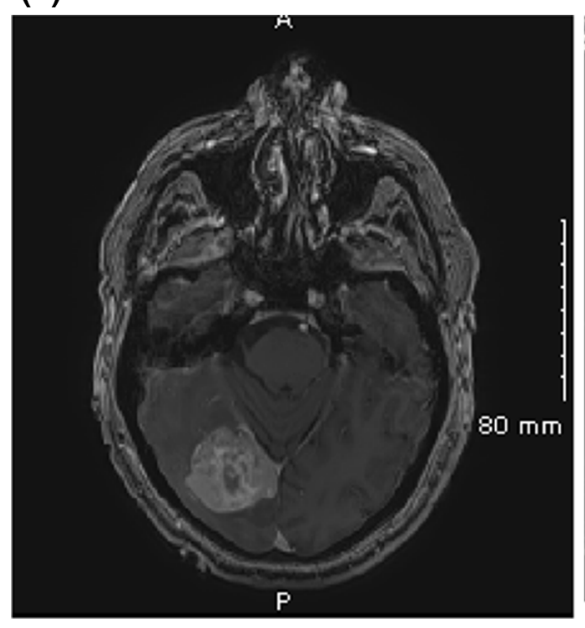

(b)

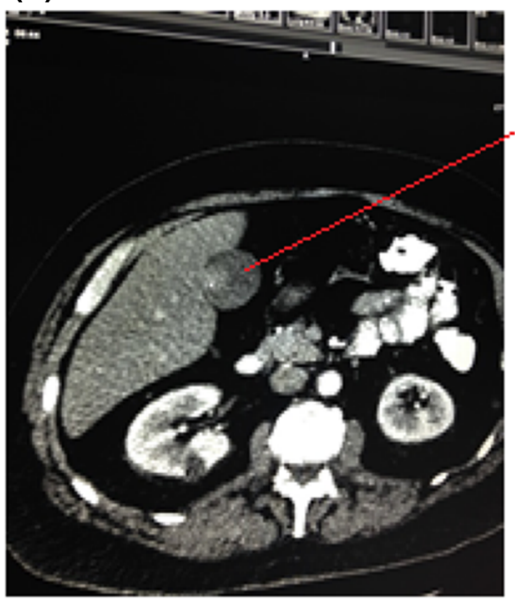

Fig. 5 a MRI brain showing RT metastatic melanoma, and $\mathbf{b}$ MRI abdomen showing mass in gall bladder (red line). MRI magnetic resonance imaging
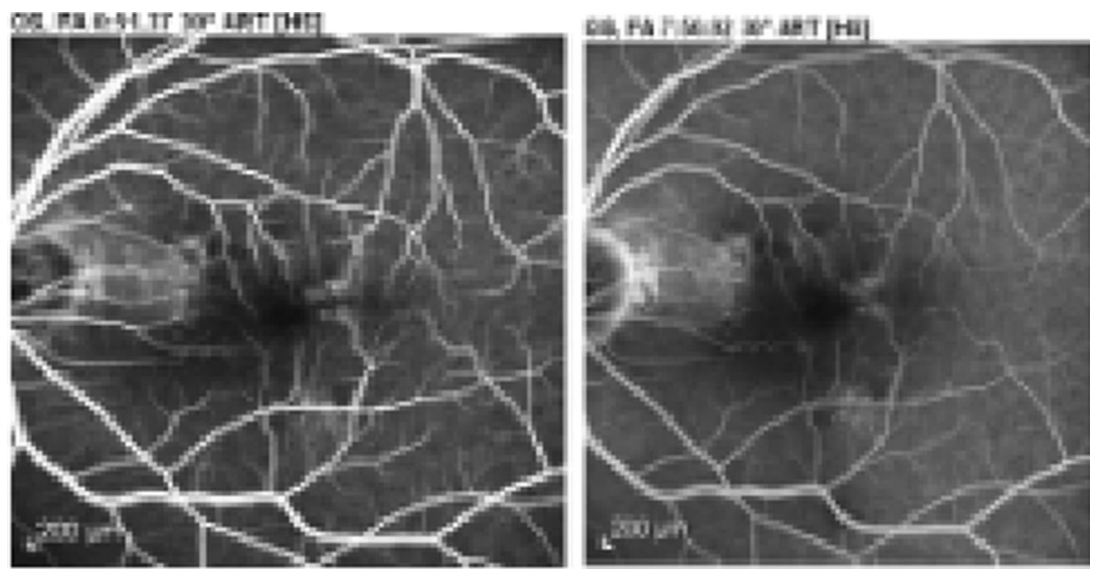

Fig. 6 Left eye fluorescein angiogram
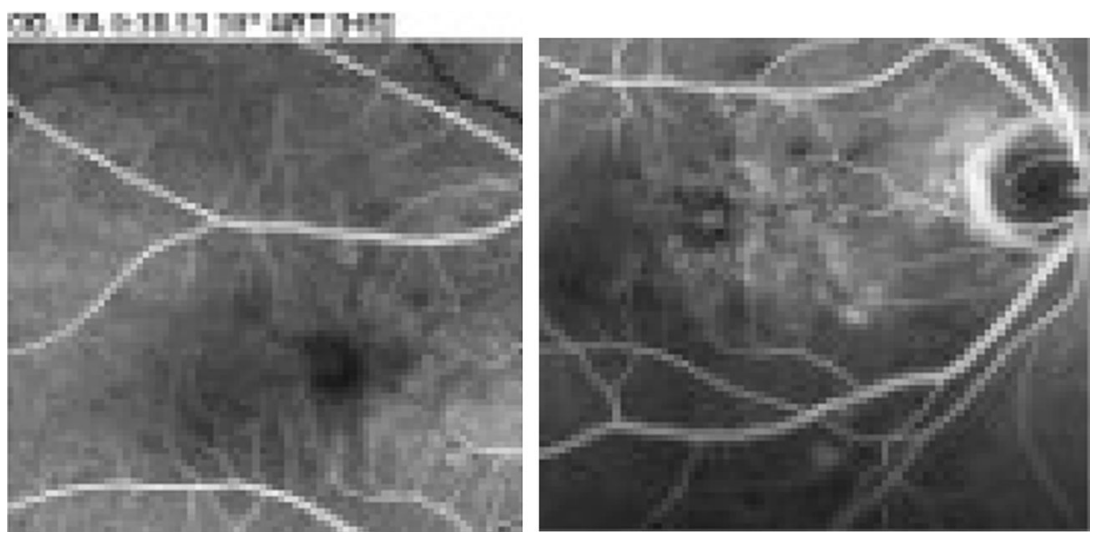

Fig. 7 Right eye fluorescein angiogram 

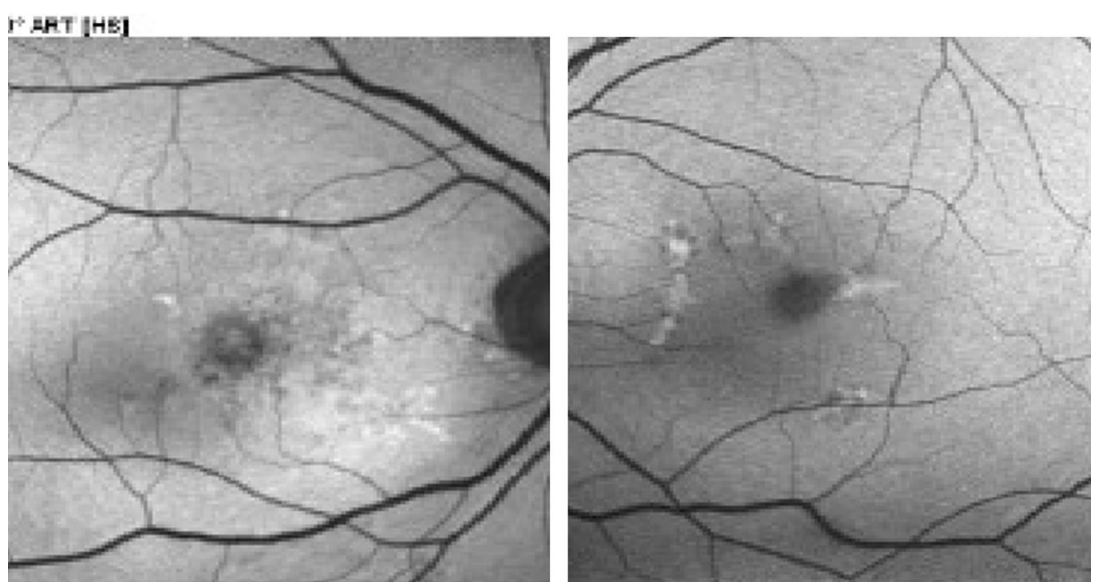

Fig. 8 Fundus autofluorescence depicting areas of hyperautofluorescence corresponding to areas of disease and detachment in both eyes

monoclonal antibody treatment such as ipilimumab revolutionized treatment strategies. Now patients are surviving longer, especially if diagnosed early [4]. Studies have shown an improved 1-year survival of $39.3 \%$ and 2-year survival rate of $24.2 \%$ [5]. Recognition of retinal findings associated with melanoma is important, especially in patients who have few symptoms such as in the patient discussed here. EPVM is pathognomonic of metastatic cancers, including melanoma. Early recognition leads to quicker identification of a primary site and appropriate expedited treatment of the disease. Unfortunately, information about eye conditions associated with metastatic melanoma patients still relies on the collection of scattered case reports [2, 3].

The retinopathy of EPVM is a paraneoplastic process, as seen in MAR, but without vascular narrowing or optic atrophy. The lesions are primarily in the maculae, and can have a similar look to the vitelliform changes seen in Best's Disease [6], in which patients have a vitelliform "yolk-like" lesion associated with a serous detachment in the macula $[7,8]$. The material of the "egg yolk" in Best's disease is believed to be lipofuscin, and is often a centralized mass within the area of detachment, while in EPVM it usually appears as small bright droplets layered in the deep retinal layers, and show bright on OCT. Patients with CSR [9] also have serous detachments, but without any "egg yolk" accumulation or discoloration; the serous detachment is secondary to choroidal vascular hyperpermeability [10-12]. In contrast to EPVM, there is also usually only one symptomatic large serous detachment centered on the macula without significant pigmentary changes, and only one eye is typically affected at a time. OCT is very useful in differentiating these three conditions.

Testing by OCT is quicker and easier for these patients, who are suddenly encompassed in care, compared to previous testing with fluorescein angiogram which required an injection. When fluorescein angiography is performed in patients with EPVM, it demonstrates some mild staining of the lesions with no leakage typically. In Best's Disease and CSR obvious hyperfluorescence and leakage is typically noted. 
In MAR where symptoms of flickering, shimmering and photopsias occur [13-16], patients with EPVM often do not present with these symptoms or any reduction of vision [17, 18]. The patient reported here did not complain of such symptoms, or of any blurring of vision. In one study only 2 out of 9 patients with cutaneous melanoma had experienced symptoms of shimmering [17]. In another study [11], two patients, both with metastatic cutaneous melanoma experienced no symptoms of shimmering in their vision. ERG had not been found to be useful in these patients as it is usually non-diagnostic or normal. This is not surprising since these patients rarely complain of light adaptation symptoms or nyctalopia. For this reason our patient did not undergo ERG testing.

Serum antibody analysis can be difficult to interpret in cases of paraneoplasia, but positive findings with any of a series of recognized retinal antigens can prompt further inquiry, even if no primary malignancy is found. Interestingly, our patient reacted with a $45 \mathrm{kd}$ retinal antigen that has been previously reported in cases of macular degeneration and CAR, and is suspected to be an example of pigment epithelium derived factor hypersensitivity [19]. Past literature reports have identified a collection of different ocular proteins involved in the paraneoplasia exhibiting a range of different molecular weights including those of 20, 22 [20], 23 [20], $40,45,47,62,120$ and $145 \mathrm{kDa}[17-21]$.

\section{CONCLUSION}

Monoclonal antibody treatment is changing survival rates in metastatic disease making early diagnosis even more important. PEVM needs to be recognized early to avoid delay in diagnosis of metastatic disease.

\section{ACKNOWLEDGMENTS}

C.E. Thirkill's research is supported by unrestricted funding from Research to Prevent Blindness, and NEI Core grant 1 P30 EY12576-09. No funding or sponsorship was received for the publication of this article. All authors had full access to all of the data in this study and take complete responsibility for the integrity of the data and accuracy of the data analysis. All named authors meet the International Committee of Medical Journal Editors (ICMJE) criteria for authorship for this manuscript, take responsibility for the integrity of the work as a whole, and have given final approval for the version to be published. Informed consent was obtained from all patients for being included in the study.

Disclosures. N. Lincoff, M. Nadeem, Z. Younus and C. E. Thirkill have nothing to disclose.

Compliance with ethics guidelines. Informed consent was given by patients.

Open Access. This article is distributed under the terms of the Creative Commons Attribution-NonCommercial 4.0 International License (http://creativecommons.org/licenses/ by-nc/4.0/), which permits any noncommercial use, distribution, and reproduction in any medium, provided you give appropriate credit to the original author(s) and the source, provide a link to the Creative Commons license, and indicate if changes were made.

\section{REFERENCES}

1. Wolchok JD, Weber JS, Maio M, et al. Four-year survival rates for patients with metastatic melanoma who received ipilimumab in phase II clinical trials. Ann Oncol. 2013;24(8):2174-80. 
2. Rosenberg C, Finger PT. Cutaneous malignant melanoma metastatic to the eye, lids, and orbit. Surv Ophthalmol. 2008;53(3):187-202.

3. Grajewski RS, Schuler-Thurner B, Mauch C, et al. Ocular diseases in metastatic cutaneous melanoma: review of 108 consecutive patients in two German tertiary centers. Graefes Arch Clin Exp Ophthalmol. 2014;252(4):679-85.

4. Queirolo P, Spagnolo F, Ascierto PA, et al. Efficacy and safety of ipilimumab in patients with advanced melanoma and brain metastases. J Neurooncol. 2014;118(1):109-16.

5. Hodi FS, O'Day SJ, McDermott DF, et al. Improved survival with ipilimumab in patients with metastatic melanoma. NEJM. 2010;363(8):711-23.

6. Vaclavik V, Ooi KG, Bird AC, Robson AG, Holder GE, Webster AR. Autofluorescence findings in acute exudative polymorphous vitelliform maculopathy. Arch Ophthalmol. 2007;125(2):274-7.

7. Querques G, Regenbogen M, Soubrane G, Souied EH. High-resolution spectral domain optical coherence tomography findings in multifocal vitelliform macular dystrophy. Surv Ophthalmol. 2009;54(2):311-6.

8. Esfahani MR, Esfahani HR, Mahmoudi A, Johari MK, Hemati K. Focal choroidal excavation in best vitelliform macular dystrophy: case report. J Clin Diagn Res. 2015;9(5):ND01-2.

9. Liegl R, Ulbig MW. Central serous chorioretinopathy. Ophthalmologica. 2014;232(2):65-76.

10. Spaide RF, Goldbaum M, Wong DW, Tang KC, Iida T. Serous detachment of the retina. Retina. 2003;23(6):820-46 (quiz 95-6).

11. Guyer DR, Yannuzzi LA, Slakter JS, Sorenson JA, Ho A, Orlock D. Digital indocyanine green videoangiography of central serous chorioretinopathy. Arch Ophthalmol. 1994;112(8): 1057-62.

12. Shin WB, Kim MK, Lee CS, Lee SC, Kim H. Comparison of the clinical manifestations between acute Vogt-Koyanagi-Harada disease and acute bilateral central serous chorioretinopathy. Korean J Ophthalmol. 2015;29(6):389-95.

13. Ikawa M, Kuriyama M. Paraneoplastic retinopathy and optic neuropathy. Brain Nerve. 2010;62(4):371-6.

14. Milam AH, Saari JC, Jacobson SG, Lubinski WP, Feun LG, Alexander KR. Autoantibodies against retinal bipolar cells in cutaneous melanoma-associated retinopathy. Invest Ophthalmol Vis Sci. 1993;34(1):91-100.

15. Alexander KR, Fishman GA, Peachey NS, Marchese $\mathrm{AL}$, Tso MO. 'On' response defect in paraneoplastic night blindness with cutaneous malignant melanoma. Invest Ophthalmol Vis Sci. 1992;33(3): 477-83.

16. Berson EL, Lessell S. Paraneoplastic night blindness with malignant melanoma. Am J Ophthalmol. 1988;106(3):307-11.

17. Rahimy E, Sarraf D. Paraneoplastic and non-paraneoplastic retinopathy and optic neuropathy: evaluation and management. Surv Ophthalmol. 2013;58(5):430-58.

18. Al-Dahmash SA, Shields CL, Bianciotto CG, Witkin AJ, Witkin SR, Shields JA. Acute exudative paraneoplastic polymorphous vitelliform maculopathy in five cases. Ophthalmic Surg Lasers Imaging. 2012;43(5):366-73.

19. Thirkill CE. Retinal pigment epithelial hypersensitivity, an association with vision loss: RPE hypersensitivity complicating paraneoplastic retinopathies. Ocul Immunol Inflamm. 2000;8(1):25-37.

20. Keltner JL, Thirkill CE, Yip PT. Clinical and immunologic characteristics of melanoma-associated retinopathy syndrome: eleven new cases and a review of 51 previously published cases. J Neuroophthalmol. 2001;21(3):173-87.

21. Modi KK, Roth DB, Green SN. Acute exudative polymorphous vitelliform maculopathy in a young man: a case report. Retin Cases Brief Rep. 2014;8(3):200-4. 